\title{
A COUNTEREXAMPLE FOR BOUNDEDNESS OF PSEUDO-DIFFERENTIAL OPERATORS ON MODULATION SPACES
}

\author{
MITSURU SUGIMOTO AND NAOHITO TOMITA
}

(Communicated by Michael T. Lacey)

\begin{abstract}
We prove that pseudo-differential operators with symbols in the class $S_{1, \delta}^{0}(0<\delta<1)$ are not always bounded on the modulation space $M^{p, q}$ $(q \neq 2)$.
\end{abstract}

\section{INTRODUCTION}

In the 1980s, by Feichtinger [2, 3], the modulation spaces $M^{p, q}$ were introduced as a fundamental function space of time-frequency analysis, which originated in signal analysis or quantum mechanics. See also [4] or Triebel [12]. The exact definition will be given in the next section, but the main idea is to consider the decaying property of a function with respect to the space variable and the variable of its Fourier transform simultaneously. Based on the same idea, Sjöstrand [9] independently introduced a symbol class which assures the $L^{2}$-boundedness of corresponding pseudo-differential operators. Since this pioneering work, the modulation spaces have also been recognized as a useful tool for the theory of pseudo-differential operators (see Gröchenig [6]).

In this paper, we investigate the boundedness property of pseudo-differential operators with symbols in $S_{\rho, \delta}^{m}$ on the modulation spaces $M^{p, q}$. There have already been several papers on this subject. For example, Gröchenig and Heil [7], Tachizawa [10], Toft [11] proved that pseudo-differential operators with symbols in $S_{0,0}^{0}$ are $M^{p, q}$-bounded. On the other hand, Calderón and Vaillancourt [1] proved that pseudo-differential operators with symbols in $S_{\delta, \delta}^{0}$ with $0<\delta<1$ (hence $S_{1, \delta}^{0}$ ) are $L^{2}$-bounded (hence $M^{2,2}$-bounded) by reducing them to the case of $S_{0,0}^{0}$. In view of these results, the class $S_{1, \delta}^{0}$ with $0<\delta<1$ appears to induce the $M^{p, q}$-boundedness as well. The objective of this paper is to show that this is not true:

Theorem 1.1. Let $1<p, q<\infty, m \in \mathbb{R}$ and $0<\delta<1$. If $m>-|1 / q-1 / 2| \delta n$, then there exists a symbol $\sigma \in S_{1, \delta}^{m}$ such that $\sigma(X, D)$ is not bounded on $M^{p, q}\left(\mathbb{R}^{n}\right)$.

In particular, Theorem 1.1 actually says that symbols in the class $S_{1, \delta}^{0}(0<\delta<1)$ do not always induce the $M^{p, q}$-boundedness in the case $q \neq 2$. We will prove this fact by constructing a counterexample.

Received by the editors January 4, 2007.

2000 Mathematics Subject Classification. Primary 42B35, 47G30.

Key words and phrases. Modulation spaces, pseudo-differential operators. 
We remark that we can admit the endpoints for $p$ and $q$ in the statement of Theorem 1.1. For example, if we assume that symbols in the class $S_{1, \delta}^{m}$ with $m>$ $-\delta n / 2$ induce the $M^{1,1}$-boundedness, then those with $m>-|1 / q-1 / 2| \delta n$ induce the $M^{q, q}$-boundedness $(1<q<2)$ by interpolating it with the boundedness of the class $S_{1, \delta}^{0}$ on $M^{2,2}=L^{2}$. This contradicts Theorem 1.1 with $1<p=q<2$; hence Theorem 1.1 is still true for $p=q=1$. This remark is due to Professor Hans G. Feichtinger.

\section{MAIN RESUlT}

Let $\mathcal{S}\left(\mathbb{R}^{n}\right)$ and $\mathcal{S}^{\prime}\left(\mathbb{R}^{n}\right)$ be the Schwartz spaces of rapidly decreasing smooth functions and tempered distributions, respectively. We define the Fourier transform $\mathcal{F} f$ and the inverse Fourier transform $\mathcal{F}^{-1} f$ of $f \in \mathcal{S}\left(\mathbb{R}^{n}\right)$ by

$$
\mathcal{F} f(\xi)=\widehat{f}(\xi)=\int_{\mathbb{R}^{n}} e^{-i \xi \cdot x} f(x) d x \quad \text { and } \quad \mathcal{F}^{-1} f(x)=\frac{1}{(2 \pi)^{n}} \int_{\mathbb{R}^{n}} e^{i x \cdot \xi} f(\xi) d \xi .
$$

Let $m \in \mathbb{R}$ and $0 \leq \delta \leq \rho \leq 1$. The symbol class $S_{\rho, \delta}^{m}$ consists of all $\sigma \in C^{\infty}\left(\mathbb{R}^{n} \times\right.$ $\mathbb{R}^{n}$ ) such that

$$
\left|\partial_{\xi}^{\alpha} \partial_{x}^{\beta} \sigma(x, \xi)\right| \leq C_{\alpha, \beta}(1+|\xi|)^{m-\rho|\alpha|+\delta|\beta|}
$$

for all $\alpha, \beta \in \mathbb{Z}_{+}^{n}=\{0,1,2, \ldots\}^{n}$. For $\sigma \in S_{\rho, \delta}^{m}$, we define the pseudo-differential operator $\sigma(X, D)$ by

$$
\sigma(X, D) f(x)=\frac{1}{(2 \pi)^{n}} \int_{\mathbb{R}^{n}} e^{i x \cdot \xi} \sigma(x, \xi) \widehat{f}(\xi) d \xi
$$

for $f \in \mathcal{S}\left(\mathbb{R}^{n}\right)$. Given a symbol $\sigma \in S_{\rho, \delta}^{m}$ with $\delta<1$, the symbol $\sigma^{*}$ defined by

$$
\begin{aligned}
\sigma^{*}(x, \xi) & =\text { Os- } \frac{1}{(2 \pi)^{n}} \int_{\mathbb{R}^{n}} \int_{\mathbb{R}^{n}} e^{-i y \cdot \zeta} \overline{\sigma(x+y, \xi+\zeta)} d y d \zeta \\
& =\lim _{\epsilon \rightarrow 0} \frac{1}{(2 \pi)^{n}} \int_{\mathbb{R}^{n}} \int_{\mathbb{R}^{n}} e^{-i y \cdot \zeta} \chi(\epsilon y, \epsilon \zeta) \overline{\sigma(x+y, \xi+\zeta)} d y d \zeta
\end{aligned}
$$

satisfies $\sigma^{*} \in S_{\rho, \delta}^{m}$ and

$$
(\sigma(X, D) f, g)=\left(f, \sigma^{*}(X, D) g\right) \quad \text { for all } f, g \in \mathcal{S}\left(\mathbb{R}^{n}\right),
$$

where $\chi \in \mathcal{S}\left(\mathbb{R}^{2 n}\right)$ satisfies $\chi(0,0)=1$ and $(\cdot, \cdot)$ denotes the inner product on $L^{2}\left(\mathbb{R}^{n}\right)$ ([8, Chapter 2, Theorem 2.6]). Note that oscillatory integrals are independent of the choice of $\chi \in \mathcal{S}\left(\mathbb{R}^{2 n}\right)$ satisfying $\chi(0,0)=1$ ([8, Chapter 1 , Theorem 6.4]).

We introduce the modulation spaces based on Gröchenig [5]. Fix a function $\gamma \in \mathcal{S}\left(\mathbb{R}^{n}\right) \backslash\{0\}$ (called a window function). Then the short-time Fourier transform $V_{\gamma} f$ of $f \in \mathcal{S}^{\prime}\left(\mathbb{R}^{n}\right)$ with respect to $\gamma$ is defined by

$$
V_{\gamma} f(x, \xi)=\left(f, M_{\xi} T_{x} \gamma\right) \quad \text { for } x, \xi \in \mathbb{R}^{n},
$$

where $M_{\xi} \gamma(t)=e^{i \xi \cdot t} \gamma(t)$ and $T_{x} \gamma(t)=\gamma(t-x)$. We can express this in a form of the integral

$$
V_{\gamma} f(x, \xi)=\int_{\mathbb{R}^{n}} f(t) \overline{\gamma(t-x)} e^{-i \xi \cdot t} d t,
$$

which actually converges for appropriate functions $f$ on $\mathbb{R}^{n}$, say $f \in L^{p}\left(\mathbb{R}^{n}\right)$. 
We note that, for $f \in \mathcal{S}^{\prime}\left(\mathbb{R}^{n}\right), V_{\gamma} f$ is continuous on $\mathbb{R}^{2 n}$ and $\left|V_{\gamma} f(x, \xi)\right| \leq$ $C(1+|x|+|\xi|)^{N}$ for some constants $C, N \geq 0([5$, Theorem 11.2.3]). Let $1 \leq$ $p, q \leq \infty$. Then the modulation space $M^{p, q}\left(\mathbb{R}^{n}\right)$ consists of all $f \in \mathcal{S}^{\prime}\left(\mathbb{R}^{n}\right)$ such that

$$
\|f\|_{M^{p, q}}=\left\|V_{\gamma} f\right\|_{L^{p, q}}=\left\{\int_{\mathbb{R}^{n}}\left(\int_{\mathbb{R}^{n}}\left|V_{\gamma} f(x, \xi)\right|^{p} d x\right)^{q / p} d \xi\right\}^{1 / q}<\infty .
$$

We note that $M^{2,2}\left(\mathbb{R}^{n}\right)=L^{2}\left(\mathbb{R}^{n}\right)$ ([5, Proposition 11.3.1]) and $M^{p, q}\left(\mathbb{R}^{n}\right)$ is a Banach space $\left(\left[5\right.\right.$, Proposition 11.3.5]). The definition of $M^{p, q}\left(\mathbb{R}^{n}\right)$ is independent of the choice of the window function $\gamma \in \mathcal{S}\left(\mathbb{R}^{n}\right) \backslash\{0\}$; that is, different window functions yield equivalent norms ([5, Proposition 11.3.2]).

We also introduce a special symbol which will act as the counterexample for the boundedness stated in the Introduction. Let $\varphi, \eta \in \mathcal{S}\left(\mathbb{R}^{n}\right)$ be real-valued functions satisfying

$$
\begin{aligned}
& \varphi: \quad \operatorname{supp} \varphi \subset\{\xi:|\xi| \leq 1 / 8\}, \quad \int_{\mathbb{R}^{n}} \varphi(\xi) d \xi=1, \\
& \eta: \quad \operatorname{supp} \eta \subset\left\{\xi: 2^{-1 / 2} \leq|\xi| \leq 2^{1 / 2}\right\}, \quad \eta=1 \text { on }\left\{\xi: 2^{-1 / 4} \leq|\xi| \leq 2^{1 / 4}\right\} .
\end{aligned}
$$

Moreover, we assume that $\varphi$ is radial. Then we define the symbol $\sigma_{\delta}$ by

$$
\sigma_{\delta}(x, \xi)=\sum_{j=j_{0}}^{\infty} 2^{j m}\left(\sum_{0<|k| \leq 2^{j \delta / 2}} e^{-i k \cdot\left(2^{j \delta / 2} x-k\right)} \Phi\left(2^{j \delta / 2} x-k\right)\right) \eta\left(2^{-j} \xi\right),
$$

where $\Phi=\mathcal{F}^{-1} \varphi, 0<\delta<1$ and $j_{0} \in \mathbb{Z}_{+}$is chosen to satisfy

$$
1+2^{j_{0}(\delta-1)+1} \leq 2^{1 / 4}, \quad 1-2^{j_{0}(\delta-1)+1} \geq 2^{-1 / 4}, \quad 2^{-j_{0} \delta / 2} \sqrt{n} \leq 2^{-3} .
$$

The symbol $\sigma_{\delta}^{*}$ is constructed from $\sigma_{\delta}$ using the oscillatory integral (2.1).

Now, we state our main result, which is a precise version of Theorem 1.1 in the Introduction.

Theorem 2.1. Let $1<p, q<\infty, 0<\delta<1$ and $m>-|1 / q-1 / 2| \delta n$. Then the symbols $\sigma_{\delta}$ and $\sigma_{\delta}^{*}$ defined by (2.3) belong to the class $S_{1, \delta}^{m}$. Moreover, if $q \geq 2$ $\left(q \leq 2\right.$ resp.), then the corresponding operator $\sigma_{\delta}(X, D)\left(\sigma_{\delta}^{*}(X, D)\right.$ resp. $)$ is not bounded on $M^{p, q}\left(\mathbb{R}^{n}\right)$.

The proof of Theorem 2.1 will be given in the next section.

\section{Proof}

In what follows, we consider the symbol $\tau_{\delta}$ instead of $\sigma_{\delta}$ for the sake of simplicity. In order to avoid confusion, we repeat the notation in this context, and also introduce a family of functions $\left\{f_{j, \epsilon, \delta}\right\}_{j}$. Let $\varphi, \psi, \eta \in \mathcal{S}\left(\mathbb{R}^{n}\right)$ be real-valued functions satisfying

$$
\begin{aligned}
& \varphi: \quad \operatorname{supp} \varphi \subset\{\xi:|\xi| \leq 1 / 8\}, \quad \int_{\mathbb{R}^{n}} \varphi(\xi) d \xi=1, \\
& \psi: \quad \operatorname{supp} \psi \subset\{\xi:|\xi| \leq 1 / 2\}, \quad \psi=1 \text { on }\{\xi:|\xi| \leq 1 / 4\}, \\
& \eta: \quad \operatorname{supp} \eta \subset\left\{\xi: 2^{-1 / 2} \leq|\xi| \leq 2^{1 / 2}\right\}, \quad \eta=1 \text { on }\left\{\xi: 2^{-1 / 4} \leq|\xi| \leq 2^{1 / 4}\right\} .
\end{aligned}
$$


Moreover, we assume that $\varphi$ and $\psi$ are radial. This assumption implies that $\Phi$ and $\Psi$ are also real-valued functions, where $\Phi=\mathcal{F}^{-1} \varphi$ and $\Psi=\mathcal{F}^{-1} \psi$. For these $\Phi, \Psi, \eta$, we define the symbol $\tau_{\delta}$ and the functions $f_{j, \epsilon, \delta}$ by

$$
\tau_{\delta}(x, \xi)=\sum_{j=j_{0}}^{\infty} 2^{j m}\left(\sum_{0<|k| \leq 2^{j \delta}} e^{-i k \cdot\left(2^{j \delta} x-k\right)} \Phi\left(2^{j \delta} x-k\right)\right) \eta\left(2^{-j} \xi\right)
$$

and

$$
f_{j, \epsilon, \delta}(x)=\sum_{0<\left|k^{\prime}\right| \leq 2^{j \delta}}\left|k^{\prime}\right|^{-n / q-\epsilon} e^{i k^{\prime} \cdot\left(x-k^{\prime}\right)} \Psi\left(x-k^{\prime}\right),
$$

where $k, k^{\prime} \in \mathbb{Z}^{n}, \epsilon>0,0<\delta<1 / 2$, and $j_{0} \in \mathbb{Z}_{+}$is chosen to satisfy

$$
1+2^{j_{0}(2 \delta-1)+1} \leq 2^{1 / 4}, \quad 1-2^{j_{0}(2 \delta-1)+1} \geq 2^{-1 / 4}, \quad 2^{-j_{0} \delta} \sqrt{n} \leq 2^{-3} .
$$

Note that $\sigma_{2 \delta}(x, \xi)=\tau_{\delta}(x, \xi)$.

Lemma 3.1. The symbol $\tau_{\delta}$ defined by (3.1) belongs to $S_{1,2 \delta}^{m}$.

Proof. Since $\operatorname{supp} \eta\left(2^{-j}.\right) \subset\left\{2^{j-1 / 2} \leq|\xi| \leq 2^{j+1 / 2}\right\}$, we see that, for each $\xi \in \mathbb{R}^{n}$, at most one term in the sum (3.1) is nonzero with respect to $j$. Note that $2^{j} \sim$ $|\xi| \sim 1+|\xi|$ on $\operatorname{supp} \eta\left(2^{-j}.\right)$. Let $\alpha, \beta \in \mathbb{Z}_{+}^{n}$ and $\xi \in \operatorname{supp} \eta\left(2^{-j} \cdot\right)$. Using

$$
\begin{aligned}
\partial_{\xi}^{\alpha} \partial_{x}^{\beta} \tau_{\delta}(x, \xi)=2^{j m} \sum_{0<|k| \leq 2^{j \delta}} \sum_{\beta_{1}+\beta_{2}=\beta} C_{\beta_{1}, \beta_{2}}\left(2^{j \delta} k\right)^{\beta_{1}} e^{-i k \cdot\left(2^{j \delta} x-k\right)} \\
\times 2^{j \delta\left|\beta_{2}\right|}\left(\partial^{\beta_{2}} \Phi\right)\left(2^{j \delta} x-k\right) 2^{-j|\alpha|}\left(\partial^{\alpha} \eta\right)\left(2^{-j} \xi\right),
\end{aligned}
$$

we have

$$
\begin{aligned}
\left|\partial_{\xi}^{\alpha} \partial_{x}^{\beta} \tau_{\delta}(x, \xi)\right| \leq C 2^{j(m-|\alpha|)}\left\|\partial^{\alpha} \eta\right\|_{L^{\infty}}\left(\sum_{0<|k| \leq 2^{j \delta}}\left(1+\left|2^{j \delta} x-k\right|\right)^{-n-1}\right) \\
\times\left(\sum_{\beta_{1}+\beta_{2}=\beta} 2^{j \delta\left(2\left|\beta_{1}\right|+\left|\beta_{2}\right|\right)}\left\|(1+|\cdot|)^{n+1}\left(\partial^{\beta_{2}} \Phi\right)\right\|_{L^{\infty}}\right) \\
\leq C 2^{j(m-|\alpha|+2 \delta|\beta|)}\left(\sup _{y \in \mathbb{R}^{n}} \sum_{k \in \mathbb{Z}^{n}}(1+|y-k|)^{-n-1}\right) \\
\leq C(1+|\xi|)^{m-|\alpha|+2 \delta|\beta|} .
\end{aligned}
$$

In the case $\xi \notin \bigcup_{j \geq j_{0}} \operatorname{supp} \eta\left(2^{-j}\right)$, we have nothing to prove.

Lemma 3.2. Let $1<p, q<\infty$, and let $f_{j, \epsilon, \delta}$ be defined by (3.2). Then the following are true:

(1) The Fourier transform of $e^{i 2^{j(1-\delta)} x_{1}} f_{j, \epsilon, \delta}(x)$ is

$$
\mathcal{F}\left[M_{2^{j(1-\delta)} e_{1}} f_{j, \epsilon, \delta}\right](\xi)=\sum_{0<\left|k^{\prime}\right| \leq 2^{j \delta}}\left|k^{\prime}\right|^{-n / q-\epsilon} e^{-i k^{\prime} \cdot\left(\xi-2^{j(1-\delta)} e_{1}\right)} \psi\left(\xi-2^{j(1-\delta)} e_{1}-k^{\prime}\right),
$$

where $x=\left(x_{1}, x_{2}, \ldots, x_{n}\right) \in \mathbb{R}^{n}$ and $e_{1}=(1,0, \ldots, 0) \in \mathbb{R}^{n}$.

(2) There exists a constant $C>0$ such that

$$
\left\|f_{j, \epsilon, \delta}\left(2^{j \delta} \cdot\right)\right\|_{M^{p, q}} \leq C 2^{j \delta n(1 / q-1)} \quad \text { for all } j \geq j_{0} .
$$


Proof. We consider only (2). Let $\gamma \in \mathcal{S}\left(\mathbb{R}^{n}\right) \backslash\{0\}$. Since

$$
\begin{aligned}
& V_{\gamma}\left[f_{j, \epsilon, \delta}\left(2^{j \delta} \cdot\right)\right]\left(2^{-j \delta} x, 2^{j \delta} \xi\right) \\
& =\sum_{0<\left|k^{\prime}\right| \leq 2^{j \delta}}\left|k^{\prime}\right|^{-n / q-\epsilon} e^{-i\left|k^{\prime}\right|^{2}} \int_{\mathbb{R}^{n}} e^{i k^{\prime} \cdot\left(2^{j \delta} t\right)} \Psi\left(2^{j \delta} t-k^{\prime}\right) \overline{\gamma\left(t-2^{-j \delta} x\right)} e^{-i\left(2^{j \delta} \xi\right) \cdot t} d t \\
& =2^{-j \delta n} \sum_{0<\left|k^{\prime}\right| \leq 2^{j \delta}}\left|k^{\prime}\right|^{-n / q-\epsilon} e^{-i\left|k^{\prime}\right|^{2}} \int_{\mathbb{R}^{n}} e^{-i\left(\xi-k^{\prime}\right) \cdot t} \Psi\left(t-k^{\prime}\right) \overline{\gamma\left(2^{-j \delta}(t-x)\right)} d t \\
& =2^{-j \delta n} \sum_{0<\left|k^{\prime}\right| \leq 2^{j \delta}}\left|k^{\prime}\right|^{-n / q-\epsilon} e^{-i\left|k^{\prime}\right|^{2}} \\
& \quad \times \int_{\mathbb{R}^{n}}\left\{\left(1+\left|\xi-k^{\prime}\right|^{2}\right)^{-n}\left(I-\Delta_{t}\right)^{n} e^{-i\left(\xi-k^{\prime}\right) \cdot t}\right\} \Psi\left(t-k^{\prime}\right) \overline{\gamma\left(2^{-j \delta}(t-x)\right)} d t,
\end{aligned}
$$

we have

$$
\begin{aligned}
\left|V_{\gamma}\left[f_{j, \epsilon, \delta}\left(2^{j \delta} \cdot\right)\right]\left(2^{-j \delta} x, 2^{j \delta} \xi\right)\right| & \leq C 2^{-j \delta n} \sum_{\left|\alpha_{1}+\alpha_{2}\right| \leq 2 n} \sum_{0<\left|k^{\prime}\right| \leq 2^{j \delta}}\left|k^{\prime}\right|^{-n / q-\epsilon} \\
\times & \left(1+\left|\xi-k^{\prime}\right|\right)^{-2 n}\left[\left|\widetilde{\partial^{\alpha_{1}} \Psi}\right| *\left|\left(\partial^{\alpha_{2}} \gamma\right)\left(2^{-j \delta} \cdot\right)\right|\right]\left(k^{\prime}-x\right),
\end{aligned}
$$

where $\widetilde{\Psi}(t)=\Psi(-t)$. Hence, we get

$$
\begin{aligned}
& \left\|V_{\gamma}\left[f_{j, \epsilon, \delta}\left(2^{j \delta} \cdot\right)\right]\right\|_{L^{p, q}} \\
& =2^{-j \delta n(1 / p-1 / q)}\left\{\int_{\mathbb{R}^{n}}\left(\int_{\mathbb{R}^{n}}\left|V_{\gamma}\left[f_{j, \epsilon, \delta}\left(2^{j \delta} \cdot\right)\right]\left(2^{-j \delta} x, 2^{j \delta} \xi\right)\right|^{p} d x\right)^{q / p} d \xi\right\}^{1 / q} \\
& \leq C 2^{-j \delta n(1 / p-1 / q+1)} \sum_{\left|\alpha_{1}+\alpha_{2}\right| \leq 2 n} \\
& \times\left\{\int_{\mathbb{R}^{n}}\left(\sum_{0<\left|k^{\prime}\right| \leq 2^{j \delta}}\left|k^{\prime}\right|^{-\frac{n}{q}-\epsilon}\left(1+\left|\xi-k^{\prime}\right|\right)^{-2 n}|| \widetilde{\partial^{\alpha_{1}} \Psi}|*|\left(\partial^{\alpha_{2}} \gamma\right)\left(2^{-j \delta} \cdot\right) \mid \|_{L^{p}}\right)^{q} d \xi\right\}^{\frac{1}{q}} \\
& \leq C 2^{-j \delta n(1 / p-1 / q+1)}\left(\sum_{\left|\alpha_{1}+\alpha_{2}\right| \leq 2 n}\left\|\widetilde{\partial^{\alpha_{1}} \Psi}\right\|_{L^{1}}\left\|\left(\partial^{\alpha_{2}} \gamma\right)\left(2^{-j \delta} \cdot\right)\right\|_{L^{p}}\right) \\
& \times\left\{\sum_{k \in \mathbb{Z}^{n}} \int_{k+[-1 / 2,1 / 2]^{n}}\left(\sum_{k^{\prime} \neq 0}\left|k^{\prime}\right|^{-n / q-\epsilon}\left(1+\left|\xi-k^{\prime}\right|\right)^{-2 n}\right)^{q} d \xi\right\}^{1 / q} \\
& \leq C 2^{j \delta n(1 / q-1)}\left\{\sum_{k \in \mathbb{Z}^{n}}\left(\sum_{k^{\prime} \neq 0}\left|k^{\prime}\right|^{-n / q-\epsilon}\left(1+\left|k-k^{\prime}\right|\right)^{-2 n}\right)^{q}\right\}^{1 / q} \leq C 2^{j \delta n(1 / q-1)} .
\end{aligned}
$$

The proof is complete.

Lemma 3.3. Let $\tau_{\delta}$ be defined by (3.1), and let

$$
g_{j, \epsilon, \delta}(x)=\sum_{0<|k|,\left|k^{\prime}\right| \leq 2^{j \delta}}\left|k^{\prime}\right|^{-n / q-\epsilon} e^{-i k \cdot(x-k)} e^{i k^{\prime} \cdot\left(x-k^{\prime}\right)} \Phi(x-k) \Psi\left(x-k^{\prime}\right) .
$$

Then

$$
\tau_{\delta}(X, D)\left[\left(M_{2^{j(1-\delta)}} e_{1} f_{j, \epsilon, \delta}\right)\left(2^{j \delta} \cdot\right)\right](x)=2^{j m} e^{i 2^{j} x_{1}} g_{j, \epsilon, \delta}\left(2^{j \delta} x\right)
$$

for all $j \geq j_{0}$ and $x \in \mathbb{R}^{n}$, where $f_{j, \epsilon, \delta}$ is defined by (3.2). 
Proof. By Lemma 3.2 (1), we have

$$
\begin{aligned}
& \mathcal{F}\left[\left(M_{2^{j(1-\delta)}} e_{1} f_{j, \epsilon, \delta}\right)\left(2^{j \delta} \cdot\right)\right](\xi)=2^{-j \delta n} \mathcal{F}\left[M_{2^{j(1-\delta)}} e_{1} f_{j, \epsilon, \delta}\right]\left(2^{-j \delta} \xi\right) \\
& =\sum_{0<\left|k^{\prime}\right| \leq 2^{j \delta}}\left|k^{\prime}\right|^{-n / q-\epsilon} e^{-i k^{\prime} \cdot\left(2^{-j \delta} \xi-2^{j(1-\delta)} e_{1}\right)} 2^{-j \delta n} \psi\left(2^{-j \delta} \xi-\left(2^{j(1-\delta)} e_{1}+k^{\prime}\right)\right) .
\end{aligned}
$$

Since $2 \delta<1, j \geq j_{0}, 1+2^{j_{0}(2 \delta-1)+1} \leq 2^{1 / 4}$ and $1-2^{j_{0}(2 \delta-1)+1} \geq 2^{-1 / 4}$, we see that

$$
\begin{aligned}
\operatorname{supp} \psi\left(2^{-j \delta} \cdot-\left(2^{j(1-\delta)} e_{1}+k^{\prime}\right)\right) & \subset\left\{\xi:\left|\xi-\left(2^{j} e_{1}+2^{j \delta} k^{\prime}\right)\right| \leq 2^{j \delta-1}\right\} \\
& \subset\left\{\xi: 2^{j}-2^{2 j \delta+1} \leq|\xi| \leq 2^{j}+2^{2 j \delta+1}\right\} \\
& \subset\left\{\xi: 2^{j-1 / 4} \leq|\xi| \leq 2^{j+1 / 4}\right\}
\end{aligned}
$$

for all $\left|k^{\prime}\right| \leq 2^{j \delta}$. This implies

$$
\operatorname{supp} \mathcal{F}\left[\left(M_{2^{j(1-\delta)} e_{1}} f_{j, \epsilon, \delta}\right)\left(2^{j \delta} \cdot\right)\right] \subset\left\{\xi: 2^{j-1 / 4} \leq|\xi| \leq 2^{j+1 / 4}\right\}
$$

for all $j \geq j_{0}$. Hence, noting that $\operatorname{supp} \eta\left(2^{-j^{\prime}} \cdot\right) \subset\left\{2^{j^{\prime}-1 / 2} \leq|\xi| \leq 2^{j^{\prime}+1 / 2}\right\}$ and $\eta\left(2^{-j^{\prime}} \cdot\right)=1$ on $\left\{2^{j^{\prime}-1 / 4} \leq|\xi| \leq 2^{j^{\prime}+1 / 4}\right\}$, we obtain

$$
\begin{aligned}
& \tau_{\delta}(X, D)\left[\left(M_{2^{j(1-\delta)} e_{1}} f_{j, \epsilon, \delta}\right)\left(2^{j \delta} \cdot\right)\right](x) \\
& =\frac{1}{(2 \pi)^{n}} \int_{\mathbb{R}^{n}} e^{i x \cdot \xi}\left\{2^{j m}\left(\sum_{0<|k| \leq 2^{j \delta}} e^{-i k \cdot\left(2^{j \delta} x-k\right)} \Phi\left(2^{j \delta} x-k\right)\right) \eta\left(2^{-j} \xi\right)\right\} \\
& \quad \times \mathcal{F}\left[\left(M_{2^{j(1-\delta)} e_{1}} f_{j, \epsilon, \delta}\right)\left(2^{j \delta} \cdot\right)\right](\xi) d \xi \\
& =2^{j m}\left(\sum_{0<|k| \leq 2^{j \delta}} e^{-i k \cdot\left(2^{j \delta} x-k\right)} \Phi\left(2^{j \delta} x-k\right)\right) \\
& \quad \times \frac{1}{(2 \pi)^{n}} \int_{\mathbb{R}^{n}} e^{i x \cdot \xi} \mathcal{F}\left[\left(M_{2^{j(1-\delta)}} e_{1} f_{j, \epsilon, \delta}\right)\left(2^{j \delta} \cdot\right)\right](\xi) d \xi \\
& =2^{j m}\left(\sum_{0<|k| \leq 2^{j \delta}} e^{-i k \cdot\left(2^{j \delta} x-k\right)} \Phi\left(2^{j \delta} x-k\right)\right)\left(M_{2^{j(1-\delta)} e_{1}} f_{j, \epsilon, \delta}\right)\left(2^{j \delta} x\right) \\
& =2^{j m} e^{i 2^{j} x_{1}} g_{j, \epsilon, \delta}\left(2^{j \delta} x\right)
\end{aligned}
$$

for all $j \geq j_{0}$ and $x \in \mathbb{R}^{n}$. The proof is complete.

Lemma 3.4 ([5, Corollary 11.2.7]). Let $f \in \mathcal{S}^{\prime}\left(\mathbb{R}^{n}\right)$ and $\gamma \in \mathcal{S}\left(\mathbb{R}^{n}\right) \backslash\{0\}$. Then

$$
(f, g)=\frac{1}{\|\gamma\|_{L^{2}}^{2}} \int_{\mathbb{R}^{2 n}} V_{\gamma} f(x, \xi) \overline{V_{\gamma} g(x, \xi)} d x d \xi \quad \text { for all } g \in \mathcal{S}\left(\mathbb{R}^{n}\right)
$$

For $1 \leq p \leq \infty, p^{\prime}$ is the conjugate exponent of $p$ (that is, $1 / p+1 / p^{\prime}=1$ ). 
Lemma 3.5 ([5, Proposition 11.3.4, Theorem 11.3.6]). If $1 \leq p, q<\infty$, then $\mathcal{S}\left(\mathbb{R}^{n}\right)$ is dense in $M^{p, q}\left(\mathbb{R}^{n}\right)$ and $M^{p, q}\left(\mathbb{R}^{n}\right)^{*}=M^{p^{\prime}, q^{\prime}}\left(\mathbb{R}^{n}\right)$ under the duality

$$
\langle f, g\rangle=\frac{1}{\|\gamma\|_{L^{2}}^{2}} \int_{\mathbb{R}^{2 n}} V_{\gamma} f(x, \xi) \overline{V_{\gamma} g(x, \xi)} d x d \xi
$$

for $f \in M^{p, q}\left(\mathbb{R}^{n}\right)$ and $g \in M^{p^{\prime}, q^{\prime}}\left(\mathbb{R}^{n}\right)$, where $\gamma \in \mathcal{S}\left(\mathbb{R}^{n}\right) \backslash\{0\}$.

We denote by $B$ the tensor product of B-splines of degree 2 ; that is,

$$
B(t)=\prod_{i=1}^{n} \chi_{[-1 / 2,1 / 2]} * \chi_{[-1 / 2,1 / 2]}\left(t_{i}\right),
$$

where $\chi_{[-1 / 2,1 / 2]}$ is the characteristic function of $[-1 / 2,1 / 2]$. Note that $\operatorname{supp} B \subset$ $[-1,1]^{n}$ and $\mathcal{F}^{-1} B(t)=(2 \pi)^{-n} \prod_{i=1}^{n}\left\{\left(\sin \left(t_{i} / 2\right)\right) /\left(t_{i} / 2\right)\right\}^{2} \in M^{p, q}\left(\mathbb{R}^{n}\right)$ for all $1 \leq$ $p, q \leq \infty$. By Lemmas 3.4 and 3.5, if $1<p, q<\infty$ and $f \in \mathcal{S}\left(\mathbb{R}^{n}\right)$, then

$$
\begin{aligned}
& \|f\|_{M^{p, q}}=\sup _{\|g\|_{M^{p^{\prime}, q^{\prime}}}=1}|\langle f, g\rangle| \geq\left|\left\langle f, \frac{\mathcal{F}^{-1} B}{\left\|\mathcal{F}^{-1} B\right\|_{M^{p^{\prime}, q^{\prime}}}}\right\rangle\right| \\
& =\frac{1}{\left\|\mathcal{F}^{-1} B\right\|_{M^{p^{\prime}, q^{\prime}}}}\left|\int_{\mathbb{R}^{2 n}} V_{\gamma} f(x, \xi) \overline{V_{\gamma}\left[\mathcal{F}^{-1} B\right](x, \xi)} d x d \xi\right| \\
& =\frac{1}{\left\|\mathcal{F}^{-1} B\right\|_{M^{p^{\prime}, q^{\prime}}}}\left|\int_{\mathbb{R}^{n}} f(t) \mathcal{F}^{-1} B(t) d t\right|,
\end{aligned}
$$

where $\gamma \in \mathcal{S}\left(\mathbb{R}^{n}\right)$ such that $\|\gamma\|_{L^{2}}=1$.

Lemma 3.6. Let $1<p, q<\infty$, and let $g_{j, \epsilon, \delta}$ be defined by (3.3). Then there exists a constant $C>0$ such that

$$
\left\|g_{j, \epsilon, \delta}\left(2^{j \delta} \cdot\right)\right\|_{M^{p, q}} \geq C 2^{-j \delta(n / q+\epsilon)} \quad \text { for all } j \geq j_{0} .
$$

Proof. Let $B$ be the tensor product of B-splines of degree 2. Note that $g_{j, \epsilon, \delta}\left(2^{j \delta} \cdot\right) \in$ $\mathcal{S}\left(\mathbb{R}^{n}\right)$. By (3.4), we have

$$
\begin{aligned}
& \left\|g_{j, \epsilon, \delta}\left(2^{j \delta} \cdot\right)\right\|_{M^{p, q}} \\
& \geq \frac{1}{\left\|\mathcal{F}^{-1} B\right\|_{M^{p^{\prime}, q^{\prime}}}}\left|\int_{\mathbb{R}^{n}} g_{j, \epsilon, \delta}\left(2^{j \delta} x\right) \mathcal{F}^{-1} B(x) d x\right| \\
& =\frac{1}{\left\|\mathcal{F}^{-1} B\right\|_{M^{p^{\prime}, q^{\prime}}}} \mid\left\{\sum_{0<|k| \leq 2^{j \delta}}|k|^{-n / q-\epsilon} \int_{\mathbb{R}^{n}} \Phi\left(2^{j \delta} x-k\right) \Psi\left(2^{j \delta} x-k\right) \mathcal{F}^{-1} B(x) d x\right\} \\
& \quad+\left\{\sum_{0<|k| \leq 2^{j \delta}} \sum_{\substack{0<\left|k^{\prime}\right| \leq 2^{j \delta} \\
k^{\prime} \neq k}}\left|k^{\prime}\right|^{-n / q-\epsilon}\right. \\
& \left.\quad \times \int_{\mathbb{R}^{n}}\left(e^{-i k \cdot\left(2^{j \delta} x-k\right)} \Phi\left(2^{j \delta} x-k\right) \mathcal{F}^{-1} B(x)\right)\left(e^{i k^{\prime} \cdot\left(2^{j \delta} x-k^{\prime}\right)} \Psi\left(2^{j \delta} x-k^{\prime}\right)\right) d x\right\} \mid \\
& =\frac{1}{\left\|\mathcal{F}^{-1} B\right\|_{M^{p^{\prime}, q^{\prime}}}}|I+I I| .
\end{aligned}
$$


We first consider $I$. Note that our assumptions $\int_{\mathbb{R}^{n}} \varphi(\xi) d \xi=1, \operatorname{supp} \varphi \subset\{|\xi| \leq$ $1 / 8\}$ and $\psi=1$ on $\{|\xi| \leq 1 / 4\}$ give $\varphi * \psi=1$ on $\{|\xi| \leq 1 / 8\}$. Since $\operatorname{supp} B \subset$ $\{|\xi| \leq \sqrt{n}\}$ and $2^{-j \delta} \sqrt{n} \leq 2^{-j_{0} \delta} \sqrt{n} \leq 1 / 8$, by Plancherel's theorem, we see that

$$
\begin{aligned}
& \sum_{0<|k| \leq 2^{j \delta}}|k|^{-n / q-\epsilon} \int_{\mathbb{R}^{n}} \Phi\left(2^{j \delta} x-k\right) \Psi\left(2^{j \delta} x-k\right) \mathcal{F}^{-1} B(x) d x \\
= & \sum_{0<|k| \leq 2^{j \delta}}|k|^{-n / q-\epsilon} \int_{\mathbb{R}^{n}}(\Phi \Psi)\left(2^{j \delta} x-k\right) \overline{\mathcal{F}^{-1} B(x)} d x \\
= & \sum_{0<|k| \leq 2^{j \delta}}|k|^{-n / q-\epsilon} \frac{1}{(2 \pi)^{2 n}} \int_{|\xi| \leq \sqrt{n}} 2^{-j \delta n} e^{-i k \cdot\left(2^{-j \delta} \xi\right)} \varphi * \psi\left(2^{-j \delta} \xi\right) \overline{B(\xi)} d \xi \\
= & C_{n} 2^{-j \delta n} \sum_{0<|k| \leq 2^{j \delta}}|k|^{-n / q-\epsilon} \int_{\mathbb{R}^{n}} e^{-i\left(2^{-j \delta} k\right) \cdot \xi} B(\xi) d \xi \\
= & C_{n} 2^{-j \delta n} \sum_{0<|k| \leq 2^{j \delta}}|k|^{-n / q-\epsilon} \prod_{i=1}^{n}\left(\frac{\sin k_{i} / 2^{j \delta+1}}{k_{i} / 2^{j \delta+1}}\right)^{2} .
\end{aligned}
$$

We next consider $I I$. Using

$$
\mathcal{F}\left[T_{k} M_{-k} \Phi\right]=e^{i|k|^{2}} T_{-k} M_{-k} \varphi \quad \text { and } \quad \mathcal{F}\left[T_{k^{\prime}} M_{-k^{\prime}} \Psi\right]=e^{i\left|k^{\prime}\right|^{2}} T_{-k^{\prime}} M_{-k^{\prime}} \psi,
$$

we have

$$
\begin{aligned}
& \int_{\mathbb{R}^{n}}\left(e^{-i k \cdot\left(2^{j \delta} x-k\right)} \Phi\left(2^{j \delta} x-k\right) \mathcal{F}^{-1} B(x)\right)\left(e^{i k^{\prime} \cdot\left(2^{j \delta} x-k^{\prime}\right)} \Psi\left(2^{j \delta} x-k^{\prime}\right)\right) d x \\
& =\int_{\mathbb{R}^{n}}\left(T_{k} M_{-k} \Phi(x) 2^{-j \delta n}\left[\mathcal{F}^{-1} B\right]\left(2^{-j \delta} x\right)\right) \overline{T_{k^{\prime}} M_{-k^{\prime}} \Psi(x)} d x \\
& =\frac{e^{i\left(|k|^{2}-\left|k^{\prime}\right|^{2}\right)}}{(2 \pi)^{2 n}} \int_{\mathbb{R}^{n}}\left[\left(T_{-k} M_{-k} \varphi\right) *\left(B\left(2^{j \delta} \cdot\right)\right)\right](\xi) \overline{\left[T_{-k^{\prime}} M_{-k^{\prime}} \psi\right](\xi)} d \xi \\
& =\frac{e^{i\left(|k|^{2}-\left|k^{\prime}\right|^{2}\right)}}{(2 \pi)^{2 n}} \int_{\mathbb{R}^{n}}\left[\left(M_{-k} \varphi\right) *\left(B\left(2^{j \delta} \cdot\right)\right)\right](\xi+k) \overline{\left[M_{-k^{\prime}} \psi\right]\left(\xi+k^{\prime}\right)} d \xi .
\end{aligned}
$$

Since $\operatorname{supp} \varphi \subset\{|\xi| \leq 1 / 8\}, \operatorname{supp} B\left(2^{j \delta} \cdot\right) \subset\left\{|\xi| \leq 2^{-j \delta} \sqrt{n}\right\} \subset\{|\xi| \leq 1 / 8\}$, we see that $\operatorname{supp}\left(M_{-k} \varphi\right) *\left(B\left(2^{j \delta} \cdot\right)\right) \subset\{|\xi| \leq 1 / 4\}$. On the other hand, $\operatorname{supp} M_{-k^{\prime}} \psi \subset$ $\{|\xi| \leq 1 / 2\}$. Hence, if $k \neq k^{\prime}$, then

$$
\frac{e^{i\left(|k|^{2}-\left|k^{\prime}\right|^{2}\right)}}{(2 \pi)^{2 n}} \int_{\mathbb{R}^{n}}\left[\left(M_{-k} \varphi\right) *\left(B\left(2^{j \delta} \cdot\right)\right)\right](\xi+k) \overline{\left[M_{-k^{\prime}} \psi\right]\left(\xi+k^{\prime}\right)} d \xi=0 ;
$$

that is, $I I=0$. Therefore, since $\prod_{i=1}^{n}\left(\sin x_{i} / x_{i}\right)^{2} \geq C$ on $[-1 / 2,1 / 2]^{n}$, we get

$$
\begin{aligned}
\frac{1}{\left\|\mathcal{F}^{-1} B\right\|_{M^{p^{\prime}, q^{\prime}}}}|I+I I| & =\left.\frac{C_{n} 2^{-j \delta n}}{\left\|\mathcal{F}^{-1} B\right\|_{M^{p^{\prime}, q^{\prime}}}}\left|\sum_{0<|k| \leq 2^{j \delta}}\right| k\right|^{-n / q-\epsilon} \prod_{i=1}^{n}\left(\frac{\sin k_{i} / 2^{j \delta+1}}{k_{i} / 2^{j \delta+1}}\right)^{2} \mid \\
& =C_{n} 2^{-j \delta n} \sum_{0<|k| \leq 2^{j \delta}}|k|^{-n / q-\epsilon} \prod_{i=1}^{n}\left(\frac{\sin k_{i} / 2^{j \delta+1}}{k_{i} / 2^{j \delta+1}}\right)^{2} \\
& \geq C_{n} 2^{-j \delta n} 2^{-j \delta(n / q+\epsilon)} \sum_{0<|k| \leq 2^{j \delta}} 1 \geq C 2^{-j \delta(n / q+\epsilon)} .
\end{aligned}
$$

The proof is complete. 
We are now ready to prove Theorem 2.1.

Proof of Theorem 2.1. Assume that $1<p, q<\infty, 0<\delta<1$ and $m>-\mid 1 / q-$ $1 / 2 \mid \delta n$. Let $\sigma_{\delta}$ be defined by $(2.3)$. Then $\sigma_{\delta}(x, \xi)=\tau_{\delta / 2}(x, \xi)$, where $\tau_{\delta / 2}$ is defined by (3.1). By Lemma 3.1, we see that $\sigma_{\delta} \in S_{1, \delta}^{m}$. This implies $\sigma_{\delta}^{*} \in S_{1, \delta}^{m}$, where $\sigma_{\delta}^{*}$ is defined by $(2.1)$.

We first consider the case $q \geq 2$. In this case, $m>(1 / q-1 / 2) \delta n$. Since $m>(1 / q-1 / 2) \delta n$, we can take $\epsilon>0$ such that $m-\epsilon \delta / 2>(1 / q-1 / 2) \delta n$. We assume that $\sigma_{\delta}(X, D)$ is bounded on $M^{p, q}\left(\mathbb{R}^{n}\right)$. Then, by Lemmas 3.2, 3.3, 3.6 and the modulation invariance of the norm $\|\cdot\|_{M^{p, q}}$, we see that

$$
\begin{aligned}
C 2^{j(m-\delta(n / q+\epsilon) / 2)} & \leq 2^{j m}\left\|g_{j, \epsilon, \delta / 2}\left(2^{j \delta / 2} \cdot\right)\right\|_{M^{p, q}}=\left\|2^{j m} e^{i 2^{j} x_{1}} g_{j, \epsilon, \delta / 2}\left(2^{j \delta / 2} \cdot\right)\right\|_{M^{p, q}} \\
& =\left\|\tau_{\delta / 2}(X, D)\left[\left(M_{2^{j(1-\delta / 2)} e_{1}} f_{j, \epsilon, \delta / 2}\right)\left(2^{j \delta / 2} \cdot\right)\right]\right\|_{M^{p, q}} \\
& =\left\|\sigma_{\delta}(X, D)\left[\left(M_{2^{j(1-\delta / 2)} e_{1}} f_{j, \epsilon, \delta / 2}\right)\left(2^{j \delta / 2} \cdot\right)\right]\right\|_{M^{p, q}} \\
& \leq\left\|\sigma_{\delta}(X, D)\right\|_{\mathcal{L}\left(M^{p, q}\right)}\left\|\left(M_{2^{j(1-\delta / 2)} e_{1}} f_{j, \epsilon, \delta / 2}\right)\left(2^{j \delta / 2} \cdot\right)\right\|_{M^{p, q}} \\
& =\left\|\sigma_{\delta}(X, D)\right\|_{\mathcal{L}\left(M^{p, q}\right)}\left\|f_{j, \epsilon, \delta / 2}\left(2^{j \delta / 2} \cdot\right)\right\|_{M^{p, q}} \leq C 2^{j \delta n(1 / q-1) / 2}
\end{aligned}
$$

for all $j \geq j_{0}$, where $f_{j, \epsilon, \delta / 2}$ and $g_{j, \epsilon, \delta / 2}$ are defined by (3.2) and (3.3). However, since $m-\epsilon \delta / 2>(1 / q-1 / 2) \delta n$, this is a contradiction. Hence, $\sigma_{\delta}$ belongs to $S_{1, \delta}^{m}$, but $\sigma_{\delta}(X, D)$ is not bounded on $M^{p, q}\left(\mathbb{R}^{n}\right)$.

We next consider the case $q \leq 2$. In this case, $m>-(1 / q-1 / 2) \delta n$. Since $q^{\prime} \geq 2$ and $m>\left(1 / q^{\prime}-1 / 2\right) \delta n$, by Theorem 2.1 with $q \geq 2$, we see that $\sigma_{\delta}(X, D)$ is not bounded on $M^{p^{\prime}, q^{\prime}}\left(\mathbb{R}^{n}\right)$. By duality and $(2.2)$, if $\sigma_{\delta}^{*}(X, D)$ is bounded on $M^{p, q}\left(\mathbb{R}^{n}\right)$, then $\sigma_{\delta}(X, D)$ is bounded on $M^{p^{\prime}, q^{\prime}}\left(\mathbb{R}^{n}\right)$. Hence, $\sigma_{\delta}^{*}$ belongs to $S_{1, \delta}^{m}$, but $\sigma_{\delta}^{*}(X, D)$ is not bounded on $M^{p, q}\left(\mathbb{R}^{n}\right)$. The proof is complete.

\section{REFERENCES}

[1] A.P. Calderón and R. Vaillancourt, A class of bounded pseudo-differential operators, Proc. Nat. Acad. Sci. U.S.A. 69 (1972), 1185-1187. MR0298480 (45:7532)

[2] H.G. Feichtinger, Banach spaces of distributions of Wiener's type and interpolation, in: P. Butzer, B. Sz.-Nagy and E. Görlich (Eds.), Proc. Conf. Oberwolfach, Functional Analysis and Approximation, August 1980, Int. Ser. Num. Math., Vol. 60, Birkhäuser-Verlag, Basel, Boston, Stuttgart, 1981, pp. 153-165. MR650272 (83g:43005)

[3] H.G. Feichtinger, Modulation spaces on locally compact abelian groups, in: M. Krishna, R. Radha and S. Thangavelu (Eds.), Wavelets and Applications, Chennai, India, Allied Publishers, New Delhi, 2003, pp. 99-140, Updated version of a technical report, University of Vienna, 1983.

[4] H.G. Feichtinger, Modulation spaces: Looking back and ahead, Sampl. Theory Signal Image Process. 5 (2006), 109-140. MR2233968 (2007j:43003)

[5] K. Gröchenig, Foundations of Time-Frequency Analysis, Birkhäuser, Boston, 2001. MR1843717 (2002h:42001)

[6] K. Gröchenig, Time-Frequency analysis of Sjöstrand's class, Rev. Mat. Iberoamericana 22 (2006), 703-724. MR2294795

[7] K. Gröchenig and C. Heil, Modulation spaces and pseudodifferential operators, Integral Equations Operator Theory 34 (1999), 439-457. MR1702232 (2001a:47051)

[8] H. Kumano-go, Pseudo-Differential Operators, MIT Press, Cambridge, 1981.

[9] J. Sjöstrand, An algebra of pseudodifferential operators, Math. Res. Lett. 1 (1994), 185-192. MR1266757 (95b:47065)

[10] K. Tachizawa, The boundedness of pseudodifferential operators on modulation spaces, Math. Nachr. 168 (1994), 263-277. MR1282643 (95h:42038) 
[11] J. Toft, Continuity properties for modulation spaces, with applications to pseudo-differential calculus. I, J. Funct. Anal. 207 (2004), 399-429. MR2032995 (2004j:35312)

[12] H. Triebel, Modulation spaces on the Euclidean $n$-space, Z. Anal. Anwendungen 2 (1983), 443-457. MR725159 (85i:46040)

Department of Mathematics, Graduate School of Science, Osaka University, ToyONAKA, OSAKA 560-0043, JAPAN

E-mail address: sugimoto@math.sci.osaka-u.ac.jp

Department of Mathematics, Graduate School of Science, Osaka University, ToyONAKA, OSAKA 560-0043, JAPAN

E-mail address: tomita@gaia.math.wani.osaka-u.ac.jp 